\title{
ANALYTICAL DYNAMIC MODEL OF COEFFICIENT OF FRICTION OF AIR PIPELINE UNDER PRESSURE
}

\author{
Vasyl DMYTRIV ${ }^{1}$, Ihor DMYTRIV ${ }^{1}$, Ivan HORODETSKYY ${ }^{2}$, Taras DMYTRIV ${ }^{3}$ \\ ${ }^{1}$ Lviv Polytechnic National University, Institute of Engineering Mechanics and Transport, Lviv, Ukraine, \\ Dmytriv_V@ukr.net \\ ${ }^{2}$ Lviv National Agrarian University, Faculty of Mechanic and Power Engineering, Lviv-Dubliany, Ukraine \\ ${ }^{3}$ Lviv Polytechnic National University, Institute of of Computer Technologies, Automation and Metrology, \\ Lviv, Ukraine
}

\begin{abstract}
To transport of the air in the pipeline, an analytical model is developed that takes into account the gas velocity, its kinematic and dynamic characteristics - density, viscosity depending on the pressure in a given space of the pipeline. The analytical model makes it possible to calculate the coefficient of friction of gas transportation in the pipeline at intervals of the absolute pressure from 220 to $2 \mathrm{kPa}$ and $\mathrm{M}<1 \mathrm{Mach}$ numbers, depending on the diameter and length of the pipeline and physical and technological characteristics of the gas. The $\mathbf{K}_{1}{ }^{*}$ aspect ratio is proposed, which characterizes in time the ratio of the dynamic force of movement of gas to the static pressure related to the diameter of the pipeline. The coefficient of air friction was modeled according to the vacuum pressure as a parameter of density and air flow. Air flow was taken from $1.917 \cdot 10^{-3} \mathrm{~m}^{3} / \mathrm{s}$ to $44.5 \cdot 10^{-3} \mathrm{~m}^{3} / \mathrm{s}$ respectively, diameters from 0.030 to $0.070 \mathrm{~m}$ and Mach number was $\mathrm{M}=0.005-0.13$. At the vacuum and excess pressures with increasing of Reynolds number and decreasing of Mach number the gas friction coefficient increased linearly. According to the simulation results as the pressure loss and the diameter of the pipeline are increased the friction coefficient increased as well. Analogically, at the vacuum metric pressure when the pressure loss and the diameter of the pipeline are increased the friction coefficient increased. At the pipeline internal diameters of 22, 30, $36 \mathrm{~mm}$ accordingly for pressure losses from 2 to $14 \mathrm{kPa}$ the coefficient of air friction varies from 0.006 to 54.527 respectively.
\end{abstract}

Keywords: coefficient of friction, Mach number, pressure, aspect ratio, Reynolds number, analytical model, vacuum.

\section{INTRODUCTION}

For the various fields of instrument-making industry and applied mechanics an actual task is to study the modes of motion of media, such as gas or fluid, which obey Newton's law (Newtonian fluids). The criterion for estimating such processes is energy loss (pressure loss), which is analyzed by the coefficients of friction of the medium, or the coefficients of friction between the layers of the medium itself.

The coefficient of friction depends on the stresses that arise among the layers of the medium in the boundary layer. Quasi-stationary stress of wall shear was obtained by calculating the (stable) coefficient of friction at the instantaneous Reynolds number and subsequently using of $\tau_{q s}=\left(\rho \cdot \lambda \cdot v^{2}\right) / 8$, where $\lambda$ is the coefficient of friction [1]. The influence of the coefficient of friction on tangential shear stresses depends on the mode of motion of the test medium, the thickness of the boundary layer, as well as the nature of the motion, which is not identical in turbulent and pulsating (forced turbulence) modes of motion [2].

As a rule, when pressure losses were studied, the empirical dependences of the friction coefficients are often used for calculations [3], which is not always consistent with the physics of the process of medium transportation.

A number of researchers worked in direction of clarification the empirical coefficients of friction were obtained experimentally for Reynolds number of specific intervals $[4,5,6,7]$. Also, the existing dependencies of the friction coefficients for liquids are used by other researchers for gases or both for two-phase flows, which does not ensure the accuracy of the calculated data [8]. According to the results of experiments and its graphical dependencies, scientists derived the empirical equations to determine the coefficients of friction over the whole interval of the Reynolds number [9]. Other researchers have approximated the experimental data to obtain an empirical equation with high accuracy for determining the friction coefficients, especially for two-phase flows (liquid and gas) $[10,11]$. The empirical dependencies of the friction coefficient approximated by the experimental data give a significant error in the calculations, which reaches up to $5 \%$ if they are determined for non-Reynolds numbers. In the case of changes in studies from liquid flows to gas, such empirical dependencies cannot be used because of changes in the physics of the process. 
Also, the coefficient of friction is calculated with using of artificial neural networks and genetic programming, with simulating the variability of the factors that characterize this coefficient $[12,13$, 14]. But the generation of factors as random numbers, may go beyond the limits what are specific to the particular process of medium transportation. Then the estimation of the coefficient of friction with the minimum error of its value does not correspond to the real process.

Therefore, for each type of transport medium, it is advisable to have an friction coefficient equation consistent with the physical processes and properties of the object.

The purpose of the research was to determine the analytical dependence of the coefficient of friction for the gas medium with the Reynolds number over the entire numerical range and to test experimentally the capacity for work of the mathematical equation

\section{DERIVING THE ANALYTICAL EQUATION FOR THE CALCULATION OF THE COEFFICIENT OF FRICTION}

Let us consider the process of air transporting in pipeline under vacuum pressure. To derive the analytical dependence of the coefficient of friction, the important factors are following the speed of movement of the air, its kinematic and dynamic characteristics - density, viscosity, pressure in the medium. Let us first evaluate analytically the air velocity in the pipeline.

To develop the model of air velocity with a vacuum in a pipeline we should consider a number of the following conditions: a) pumping the air, and therefore air motion in the pipeline is forced and depends on the vacuum pressure and the productivity of the vacuum pump; b) quantity of air intake into the pipeline is variable over the length of the pipeline; c) air intake into the pipeline is subject to the amplitude-frequency characteristic, the amplitude and frequency of which is identical to the technological parameters of the operation of the given equipment; $d$ ) in the pipeline is the pressure drop and its gradient on the pipeline sections is different.

We consider air as an uncompressed medium with velocity no more than $100 \mathrm{~m} / \mathrm{s}$. In other cases, it is necessary to take into account the dependence of the air density $\rho_{\text {air }}$ on the pressure $p_{V}$, which depends on the velocity [15].

Let us consider the physical process of pressure drop on the pipeline segment of $d x$ length (fig. 1). Let in the point of 1 is the $p_{1}$ pressure and the $v_{1}$ air velocity. At point of $2-p_{2}$ pressure and $v_{2}$ air velocity. The cross-sectional area of the pipeline is $S$.

Considering the continuity equation of air motion, we write:

$$
\rho_{1} \cdot v_{1} \cdot S=\rho_{2} \cdot v_{2} \cdot S
$$

The right or left sides of equation of (1) are the loss at the given $d x$ section of the pipeline:

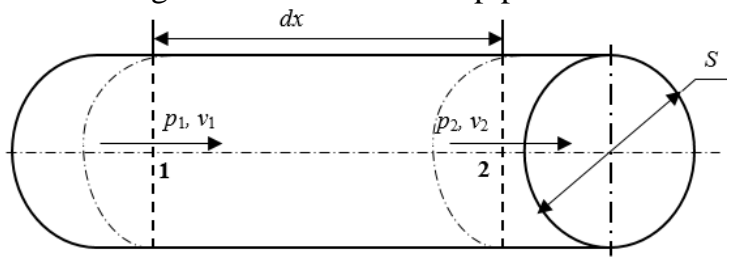

Fig. 1. Scheme of calculation of pressure loss

$$
m=\rho \cdot v \cdot S .
$$

According to the Clapeyron equation, in the given volume of pipeline the amount of air is calculated by the equation of (3)

$$
m=\frac{M_{\pi} \cdot p \cdot V}{R_{0} \cdot T},
$$

where $M_{\Omega}$ - the molar mass of air, $\mathrm{kg} / \mathrm{mole}$;

$p$ - the pressure, $\mathrm{Pa}$;

$V$ - the volume of air in a given length of pipeline, $\mathrm{m}^{3}$;

$R_{0}$ - the absolute gas constant, $\mathrm{kg} \cdot \mathrm{m}^{2} /\left(\right.$ mole $\left.\cdot \mathrm{K} \cdot \mathrm{s}^{2}\right)$;

$T$ - the air temperature, $\mathrm{K}$.

In the (3) equation we changed the $K_{1}=\frac{M_{l}}{R_{0} \cdot T}$, and the volume of $V=v \cdot S$.

The (3) equation with changes was inserted into equation of (2) and we got

$$
\rho \cdot v \cdot S=K_{1} \cdot p \cdot v \cdot S .
$$

We differentiate the right and left sides of (4) equation by $x$, taking into account that $\mathrm{S}$ - the crosssectional area of the pipeline is constant:

$$
\frac{d}{d x}(\rho \cdot v)=K_{1} \cdot \frac{d}{d x}(p \cdot v) .
$$

The equation is obtained

$$
\frac{v \cdot d \rho}{d x}+\frac{\rho \cdot d v}{d x}=K_{1} \cdot\left(\frac{v \cdot d p}{d x}+\frac{p \cdot d v}{d x}\right) .
$$

From the Euler equation [15] we define the component of $d v / d x$

$$
\frac{d v}{d x}=-\frac{1}{v \cdot \rho} \cdot \frac{d p}{d x}
$$

and after it insert into (6) equation, we will obtain the (8) equation:

$$
\frac{v \cdot d \rho}{d x}-\frac{1}{v} \cdot \frac{d p}{d x}=K_{1} \cdot\left(\frac{v \cdot d p}{d x}-\frac{p}{\rho \cdot v} \cdot \frac{d p}{d x}\right)
$$

With take into account the pressure loss for $\sqrt{K_{1}} \cdot \frac{\lambda}{D} \cdot \frac{v^{2}}{2} \cdot \rho$ viscous friction overcoming the (8) equation will have a view of (9):

$$
\begin{aligned}
& \frac{v \cdot d \rho}{d x}=\frac{K_{1} \cdot v \cdot d p}{d x}-\frac{K_{1} \cdot p}{\rho \cdot v} \cdot \frac{d p}{d x}+ \\
& +\frac{1}{v} \cdot \frac{d p}{d x}-\sqrt{K_{1}} \cdot \frac{\lambda}{D} \cdot \frac{v^{2}}{2} \cdot \rho
\end{aligned},
$$

After product of (9) equation on $d x / d \rho$ and simplification we will have the (10) equation: 


$$
\begin{aligned}
& v+\frac{K_{1} \cdot p}{\rho \cdot v} \cdot \frac{d p}{d \rho}=\frac{K_{1} \cdot v \cdot d p}{d \rho}+ \\
& +\frac{1}{v} \cdot \frac{d p}{d \rho}-\sqrt{K_{1}} \cdot \frac{\lambda}{D} \cdot \frac{v^{2}}{2} \cdot \rho \cdot \frac{d x}{d \rho}
\end{aligned},
$$

where $D$ - the diameter of pipeline, $\mathrm{m}$.

We use the dependence of the speed of sound in the medium as a ratio of pressure to the density of the medium [15]:

$$
a^{2}=\frac{d p}{d \rho}=\frac{p}{\rho},
$$

we changed the (10) equation with took into account the (1) equation and we obtained the (12) equation:

$$
\begin{aligned}
& v \cdot\left(1-K_{1} \cdot a^{2}\right)+\frac{a^{2}}{v} \cdot\left(K_{1} \cdot a^{2}-1\right)= \\
& =-\sqrt{K_{1}} \cdot \frac{\lambda}{D} \cdot \frac{v^{2}}{2} \cdot \rho \cdot \frac{d x}{d \rho}
\end{aligned}
$$

To develop the analytical dependence of the $\lambda$ air friction coefficient, we introduce the $M$ Mach number by dividing both parts of (12) equation by $a^{2} / v$. Then the (12) equation takes the view of (13) equation:

or

$$
\begin{aligned}
& \frac{v^{2}}{a^{2}} \cdot\left(1-K_{1} \cdot a^{2}\right)+\left(K_{1} \cdot a^{2}-1\right)= \\
& =-\sqrt{K_{1}} \cdot \frac{\lambda}{D} \cdot \frac{v^{2}}{a^{2}} \cdot \frac{\rho}{2} \cdot v \cdot \frac{d x}{d \rho}
\end{aligned}
$$

$$
\begin{aligned}
& M^{2} \cdot\left(1-K_{1} \cdot a^{2}\right)-\left(1-K_{1} \cdot a^{2}\right)= \\
& =-\sqrt{K_{1}} \cdot \frac{\lambda}{D} \cdot M^{2} \cdot \frac{\rho}{2} \cdot v \cdot \frac{d x}{d \rho}
\end{aligned}
$$

For convenience of integration we transform the (14) dependence to the form of (15) equation:

$$
\begin{aligned}
& \frac{1-M^{2}}{M^{2}} \cdot\left(1-K_{1} \cdot a^{2}\right) \cdot 2 \cdot D \cdot \frac{d \rho}{\rho}= \\
& =\sqrt{K_{1}} \cdot v \cdot \lambda \cdot d x
\end{aligned}
$$

We change the $\sqrt{K_{1}} \cdot v$ with take into account the (2) and (4) equations:

$$
\sqrt{K_{1}} \cdot v=\sqrt{\frac{m \cdot v^{2}}{p \cdot v \cdot S}}=\sqrt{\frac{m \cdot v}{p \cdot S}}=\sqrt{K_{1}^{*}} .
$$

Than the (16) equation will have the view of (17) equation:

$$
\frac{1-M^{2}}{M^{2}} \cdot \frac{2 \cdot D}{\sqrt{K_{1}^{*}}} \cdot\left(1-K_{1} \cdot a^{2}\right) \cdot \frac{d \rho}{\rho}=\lambda \cdot d x .
$$

We obtain the (18) equation after the $K_{1}$ coefficient was changed on equation of $K_{1}=m /(p \cdot V)$ and $a^{2}$ on $a^{2}=p / \rho$ :

$$
\frac{1-M^{2}}{M^{2}} \cdot \frac{2 \cdot D}{\sqrt{K_{1}^{*}}} \cdot\left(1-\frac{m}{V \cdot \rho}\right) \cdot \frac{d \rho}{\rho}=\lambda \cdot d x .
$$

We integrate the (18) equation with the limits of $\rho-$ from $\rho_{B}$ to $\rho$ and for $x$ - from 0 to $x$ :

$$
\frac{1-M^{2}}{M^{2}} \cdot \frac{2 \cdot D}{\sqrt{K_{1}^{*}}} \cdot\left(\int_{\rho_{B}}^{\rho} \frac{d \rho}{\rho}-\frac{m}{V} \cdot \int_{\rho_{B}}^{\rho} \frac{d \rho}{\rho^{2}}\right)=\lambda \cdot \int_{0}^{x} d x \cdot
$$

Than after integration the (19) equation will have the view of (20) equation:

$$
\lambda=\frac{1-M^{2}}{M^{2}} \cdot \frac{2 \cdot D}{x \cdot \sqrt{K_{1}^{*}}} \cdot\left(\ln \frac{\rho}{\rho_{B}}-\frac{m}{V} \cdot\left(\frac{1}{\rho_{B}}-\frac{1}{\rho}\right)\right),
$$

where: $M$ - the Mach number;

$D$ - the inner diameter of pipeline, $\mathrm{m}$;

$x$ - the length of the pipeline section for which the coefficient of air friction is calculated, $\mathrm{m}$;

$\rho$ - the air density at given pressure, $\mathrm{kg} / \mathrm{m}^{3}$;

$\rho_{B}-$ the air density at pressure of vacuum of $50 \mathrm{kPa}$;

$m$ - the mass flow of air in the pipeline, reduced to atmospheric pressure, $\mathrm{kg} / \mathrm{s}$;

$V$ - the volume flow of air at a given pressure, $\mathrm{m}^{3} / \mathrm{s}$; $K_{1}^{*}$ - the aspect ratio characterizes the ratio of forces of (16) equation.

\section{SIMULATION OF COEFFICIENT OF FRICTION}

The coefficient of air friction was modeled according to the vacuum pressure as a parameter of density and air flow. Air flow was taken from $2.951 \cdot 10^{-3} \mathrm{~m}^{3} / \mathrm{s}$ to $44.5 \cdot 10^{-3} \mathrm{~m}^{3} / \mathrm{s}$ respectively, diameter from 0.030 to $0.070 \mathrm{~m}$ and Mach number was $M=0.005-0.13$. The simulation results for vacuum changes from $100 \mathrm{kPa}$ to $2 \mathrm{kPa}$ are shown on Fig. 2 and 3 , the axis of the $\lambda$ coefficient of friction is given as a logarithmic scale.

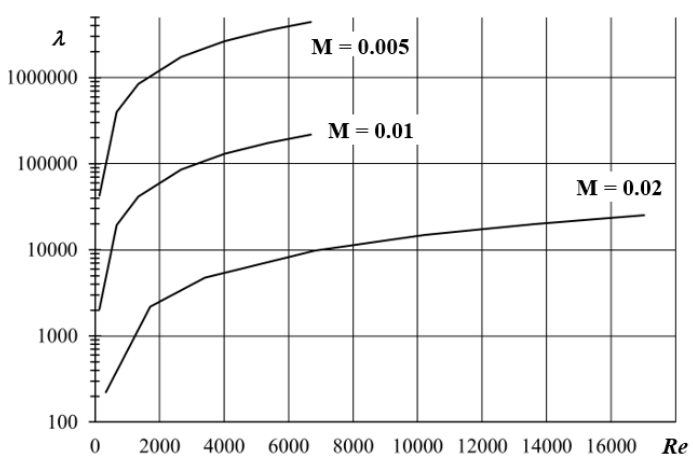

Fig. 2. Variation of $\lambda$ coefficient of friction with $R e$ Reynolds number within the interval of Mach number of $\mathrm{M}=0.005-0.02$ at the limits of absolute pressure (vacuum) from 100 to $2 \mathrm{kPa}$

The coefficient of friction was increased as the Reynolds number was increased and the Mach number was decreased.

For better perception, the simulation results are presented in a 3D coordinate system. The simulation parameters were the real modes of operation of milking machines, which made it possible to carry out the planned experiments with using the technological equipment. For analytical modelling, the airflow rate was from 0.00575 to 
$0.01534 \mathrm{~m}^{3} / \mathrm{s}$, pressure losses are within $2-14 \mathrm{kPa}$ with the nominal vacuum pressure of $50 \mathrm{kPa}$. The pipeline section was $L=37 \mathrm{~m}$ long. The simulation results are shown on fig. 4 .

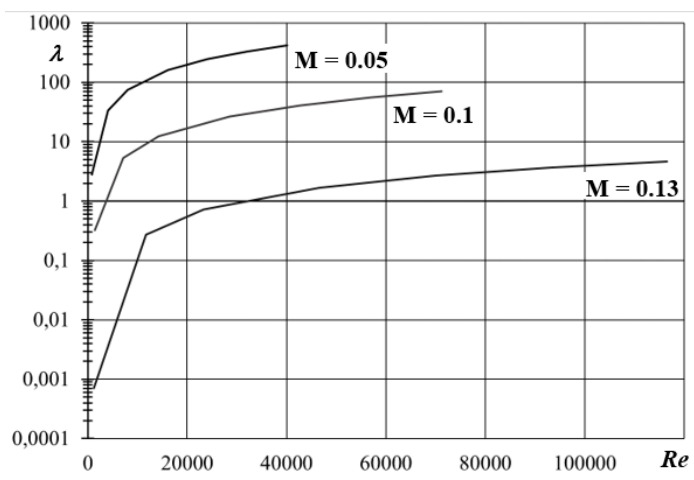

Fig. 3. Variation of $\lambda$ coefficient of friction with $R e$ Reynolds number within the interval of Mach number of $M=0.05-0.13$ at the limits of absolute pressure (vacuum) from 100 to $2 \mathrm{kPa}$

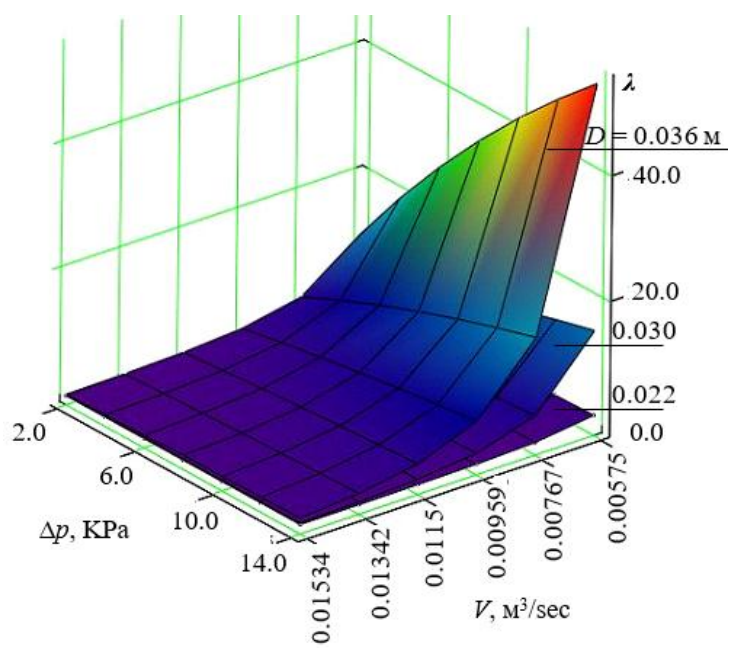

Fig. 4. Variation of $\lambda$ coefficient of air friction from the $\Delta p$ pressure lost and the $V$ airflow with the given $D$ diameter of pipeline

The friction coefficient increases with the increase of pressure losses as with the increase of the diameter of the pipeline.

At the pipeline diameters of 25.4, 34 and $40 \mathrm{~mm}$ (internal diameter $22,30,36 \mathrm{~mm}$ ), the coefficient of air friction (Fig. 4) varies from 0.006 to 54.527, accordingly for pressure losses from 2 to $14 \mathrm{kPa}$.

The fig. 5 shows the results of modelling the coefficient of friction for air flow rates within $0.01534-0.03080 \mathrm{~m}^{3} / \mathrm{s}$, pressure losses within 2 $14 \mathrm{kPa}$ and the $L=22 \mathrm{~m}$ length of the pipeline section.

Accordingly, at the pipeline diameters of 40, 50 and $60 \mathrm{~mm}$ (internal diameter 36, 46 and $56 \mathrm{~mm}$ ), the coefficient of air friction (Fig. 5) varied from 0.009 to 8.641 at pressure losses from 2 to $14 \mathrm{kPa}$.

The simulations of the coefficient of friction of the air at excess pressure within $100-220 \mathrm{kPa}$ are shown on 6 and 7 Figures. Air consumption was taken from $2.987 \cdot 10^{-3} \mathrm{~m}^{3} / \mathrm{s}$ to $33.759 \cdot 10^{-3} \mathrm{~m}^{3} / \mathrm{s}$, respectively, the diameters were $0.036-0.070 \mathrm{~m}$ and Mach number - within of $M=0.005-0.13$. The axis of the $\lambda$ friction coefficient was presented in the form of a logarithmic scale (figs. 6 and 7).

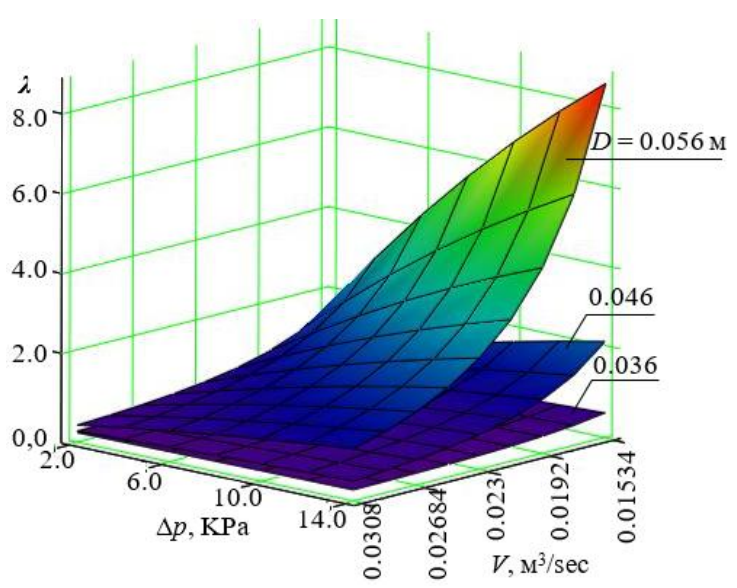

Fig. 5. Variation of $\lambda$ coefficient of air friction from the $\Delta p$ pressure lost and the $V$ airflow with the given $D$ diameter of pipeline

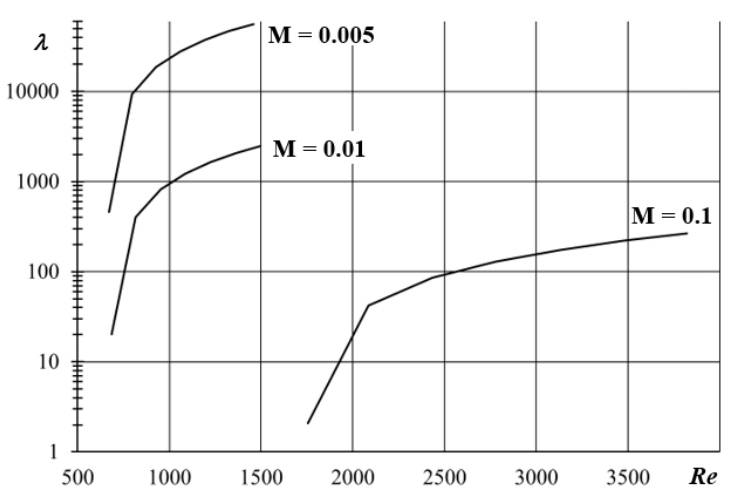

Fig. 6. Variation of $\lambda$ coefficient of air friction from the $R e$ Reynolds number within the interval of Mach number of $M=0.005-0.02$ at the limits of absolute pressure from 100 to $220 \mathrm{kPa}$

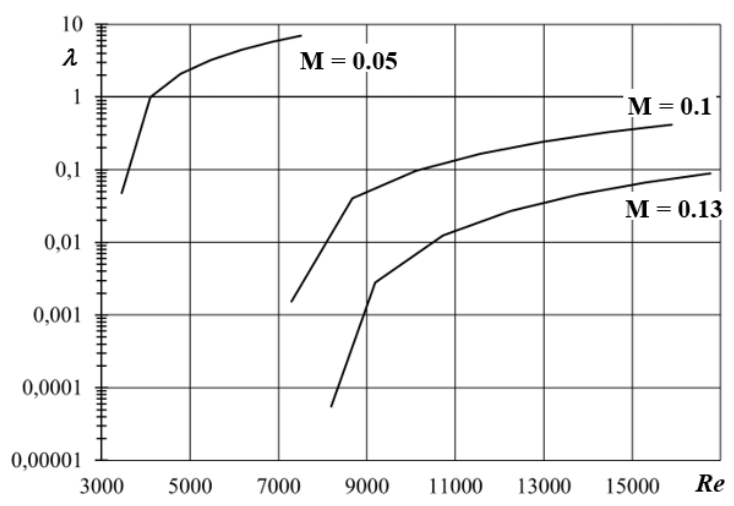

Fig. 7. Variation of $\lambda$ coefficient of air friction from the $R e$ Reynolds number within the interval of Mach number of $M=0.05-0.13$ at the limits of absolute pressure from 100 to $220 \mathrm{kPa}$

Similarly to vacuum, with the excess pressure in the pipeline the friction coefficient was increased as 
the Reynolds number was increased and the Mach number was decreased.

For Mach number of $M=0.005$, under vacuum at air flow of $V=5.751 \cdot 10^{-3} \mathrm{~m}^{3} / \mathrm{s}$ and Reynolds numbers of $R e=6694-133.88$, the friction coefficient was of $\lambda=444540-42702$ respectively, the velocity of air flow was of $v=1.497 \mathrm{~m} / \mathrm{s}$, the aspect ratio was of $K_{1}^{*}=0.0799$. At excess pressure with Mach number of $M=0.005$, these parameters were: $V=5.681 \cdot 10^{-3} \mathrm{~m}^{3} / \mathrm{s}, \quad R e=669.72-145.798$, $\lambda=461.75-56184.4, \quad v=1.469 \mathrm{~m} / \mathrm{s}, \quad K_{1}^{*}=0.0794$ respectively.

Let us consider the results for other Mach numbers. For Mach number of $M=0.01$, under vacuum: $V=2.951 \cdot 10^{-3} \mathrm{~m}^{3} / \mathrm{s}, \quad R e=6618-133.975$, $\lambda=219236-2051, \quad v=2.9 \mathrm{~m} / \mathrm{s}, \quad K_{1}^{*}=0.11163$ respectively; at excess pressure: $V=2.987$. $10^{-3} \mathrm{~m}^{3} / \mathrm{s}, \operatorname{Re}=686.58-1495.53, \lambda=20.1-2482.29$, $v=2.936 \mathrm{~m} / \mathrm{s}, \quad K_{1}^{*}=0.1123$ respectively.

For Mach number of $M=0.1$, under vacuum: $V=33.375 \cdot 10^{-3} \mathrm{~m}^{3} / \mathrm{s}, R e=71243-1424, \lambda=70.5-$ $0.324 v=29.01 \mathrm{~m} / \mathrm{s}, \quad K_{1}^{*}=0.353$ respectively; at excess pressure: $V=33.759 \cdot 10^{-3} \mathrm{~m}^{3} / \mathrm{s}, R e=7298.33$ $-15897.36, \lambda=0.001533-0.41796, \quad v=29.348$ $\mathrm{m} / \mathrm{s}, K_{1}^{*}=0.3551$ respectively.

For Mach number of $M=0.2$, under vacuum: $V=44.5 \cdot 10^{-3} \mathrm{~m}^{3} / \mathrm{s}, R e=116546-1165, \lambda=4.6777-$ $0.00071, v=58.235 \mathrm{~m} / \mathrm{s}, \quad K_{1}^{*}=0.5$ respectively; at excess pressure: $V=44.583 \cdot 10^{-3} \mathrm{~m}^{3} / \mathrm{s}, \quad R e=$ $11897.15-25796.79, \quad \lambda=4 \cdot 10^{-6}-0.03273, \quad v=$ $58.697 \mathrm{~m} / \mathrm{s}, K_{1}^{*}=0.502$ respectively.

\section{CONCLUSIONS}

The analysis of the simulation results shows that with vacuum and excess pressures, with increasing of Reynolds number and decreasing of Mach number, the gas friction coefficient increased linearly. As the pressure loss and the diameter of the pipeline are increased the friction coefficient increased as well.

An increase in the Reynolds number characterizes an increase follows: the dynamic parameters of gas flow, velocity and flow modes from laminar to turbulent, which consequently leads to an increase in Mach number. As the Reynolds number increased, the density of gas in the pipeline increased, both under vacuum and excess pressures in the medium under study. With increase of gas density, the gas viscosity increases, thereby the tangential stresses increase and lead to pressure losses, which in turn characterize the increase of the friction coefficient of the air as it moves in the pipeline.

The developed analytical dependence of the coefficient of resistance characterizes the aforesaid logic of the physics of the mediums flow process of pneumatic systems.
It should be noted that the developed analytical dependence works for Newtonian media and $M<1$ Mach numbers, the gas flow is caused by the pressure difference in the space with design parameters of reduced diameter and length of transportation.

The criterion for the estimation of the flow dynamics the $K_{1}^{*}$ aspect ratio is proposed, which characterizes the ratio of the dynamic force of movement of gas to the static pressure related to the diameter of the pipeline. As the $K_{1}^{*}$ aspect ratio is increased, the $\lambda$ coefficient of friction for the gaseous medium is decreased.

The developed analytical dependence of the coefficient of friction can be applied to the design of pipe line transport of gas medium and pneumodynamic technological systems under vacuum and excess pressures in the system.

\section{REFERENCES}

1. He S, Ariyaratne C. Wall shear stress in the early stage of unsteady turbulent pipe flow. Journal of Hydraulic Engineering. 2011; 137(5): 606-610.

2. Sundstrom LRJ, Cervantes MJ. On the similarity of pulsating and accelerating turbulent pipe flows. Flow, Turbulence and Combustion. 2018; 100(2): 417-436. https://doi.org/10.1007/s10494-0179855-5.

3. Kong R, Kim S. Characterization of horizontal airwater two-phase flow. The 16th International Topical Meeting on Nuclear Reactor Thermal Hydraulics (NURETH-16), August 30-September 4, Chicago, USA. 2015: 5559-5572.

4. Offor UH, Alabi SB. An accurate and computationally efficient explicit friction factor model. Advances in Chemical Engineering and Science. 2016; 6: 237-245. https://dx.doi.org/10.4236/aces.2016.63024.

5. Medina YC, Fonticiella OMC, Morales OFG. Design and modelation of piping systems by means of use friction factor in the transition turbulent zone. Mathematical Modelling of Engineering Problems. 2017; 4(4): 162-167.

https://dx.doi.org/10.18280/mmep.040404.

6. Azizi N, Homayoon R, Hojjati MR. Predicting the Colebrook-White friction factor in the pipe flow by new explicit correlations. Journal of Fluids Engineering. 2018; 141(5).

https://dx.doi.org/10.1115/1.4041232.

7. Pimenta BD, Robaina AD, Peiter MX, Mezzomo W, Kirchner JH, Ben LHB. Performance of explicit approximations of the coefficient of head loss for pressurized conduits. Brazilian Journal of Agricultural and Environmental Engineering (Revista Brasileira de Engenharia Agrícola e Ambiental). 2018; 22(5): 301-307.

8. Tarek A, Ganat and Meftah Hrairi. Gas-liquid twophase upward flow through a vertical pipe: influence of pressure drop on the measurement of fluid flow rate. Energies. MDPI, Open Access Journal. 2018; 11(11):1-23. https://dx.doi.org/10.3390/en11112937.

9. Brkic D, Praks P. Unified friction formulation from laminar to fully rough turbulent flow. Applied 
Sciences. 2018; 8(11): 2036.

https://dx.doi.org/10.3390/app8112036.

10. Ortiz-Vidal LE, Mureithi N, Rodriguez OMH. Friction factor in two-phase gas-liquid pipe flow. 8-th International Conference on Multiphase Flow ICMF-2013, May 26-31. Jeju, Korea 2013.

11. Lukman S, Oke IA. Accurate solutions of colebrookwhite's friction factor formulae. Nigerian Journal of Technology (NIJOTECH). Nigeria. 2017; 36(4): 1039-1048.

12. Salmasi F, Khatibi R, Ghorbani MA. A study of friction factor formulation in pipes using artificial intelligence techniques and explicit equations. Turkish Journal of Engineering and Environmental Sciences. 2012; 36(2): 121-138.

13. Offor UH, Alabi SB. Artificial neural network model for friction factor prediction. Journal of Materials Science and Chemical Engineering. 2016; 4: 77-83.

14. Brkic D, Sojbasic C. Intelligent Flow Friction Estimation. Computational Intelligence and Neuroscience. 2016.

https://dx.doi.org/10.1155/2016/5242596.

15. Loitsianskyi LG. Mechanics of fluid and gas. State Publishing House of technical and theoretical literature. Moscow, Russia. 1952

Received 2019-08-20

Accepted 2019-11-18

Available online 2019-11-20

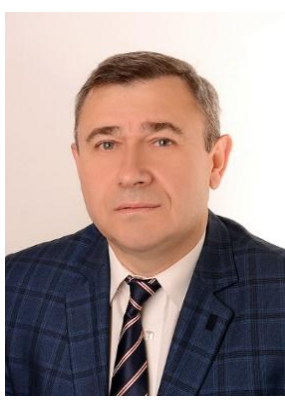

Vasyl DMYTRIV, Doctor of Technical Sciences (DSc. Eng.), Associate Professor, Professor of Department of Mechanics and Automation of Mechanical Engineering, Lviv Polytechnic National University, Ukraine. In scientific work conducts research in the dynamics, synthesis and optimization of hydropneumodynamic, vibration and adaptive cyber-physical systems of automated production processes. Engaged in problems of dynamics, strength and technical diagnostics of machines. Author and co-author of more than 350 scientific publications, including 7 monographs, 14 manuals and 36 patents.

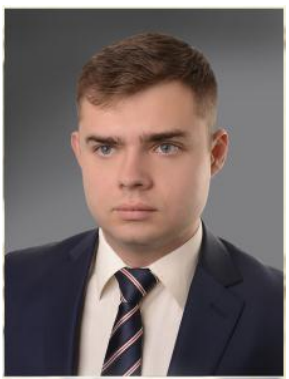

Ihor DMYTRIV, Candidate of Technical Sciences (Ph.D), Associate Professor Department of Automobile Technology Operation and Repair, Lviv Polytechnic National University, Ukraine. The areas of research are mechanization, automation and robotization of technological processes, technologies and tools of computer diagnostics of internal combustion engine, theory of planned factor experiment, software and hardware of scientific research. Author and co-author of more than 90 publications, including 2 monographs, 2 manuals and a laboratory workshop, 6 patents.

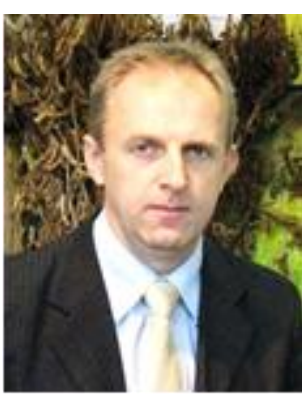

Ivan HORODETSKYY, Candidate of Technical Sciences $(\mathrm{PhD})$, Associated Professor, Department of Occupational Safety and Project Management, Lviv National Agrarian University, Dubliany, Ukraine. Field of research: study and simulation of fault development; develop the analytical methods of safety of technical and human-machine systems; designing of preventing technical and managerial measures, research and production of fan jet nozzle. Author and co-author of more than 70 scientific publications. 3 monographs, 5 manuals, 3 patents.

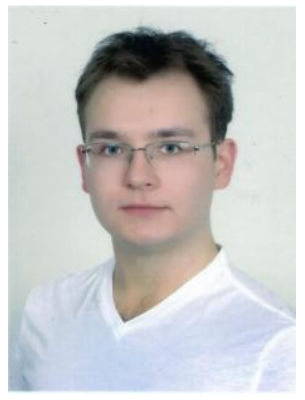

Taras DMYTRIV, Student Department of Information Measurement Technologies, Lviv Polytechnic National University, Ukraine. Range of scientific interests - theory and programming of informationmeasuring systems for diagnostics of technical systems and machines. The co-author of 5 scientific publications. 